\title{
Rofecoxib caused fewer endoscopic gastroduodenal ulcers than ibuprofen in osteoarthritis
}

\author{
Laine L, Harper S, Simon T, et al, for the Rofecoxib Osteoarthritis Endoscopy Study Group. A randomized trial comparing the \\ effect of rofecoxib, a cyclooxygenase 2-specific inhibitor, with that of ibuprofen on the gastroduodenal mucosa of patients \\ with osteoarthritis. Gastroenterology 1999 Oct;117:776-83.
}

QUESTION: In patients with osteoarthritis (OA), does rofecoxib at doses of 25 and $50 \mathrm{mg} /$ day cause fewer endoscopic gastroduodenal ulcers than ibuprofen?

Design

Randomised (allocation concealed*), blinded (patients, clinicians, and outcome assessors),* placebo controlled trial with 24 week follow up.

Setting

33 clinical centres in the United States.

Source of funding: Merck E Co.,Inc.

For correspondence: Dr L Laine, GI Liver Division, Department of Medicine (LAC

12-137), University of

Southern California

School of Medicine,

2025 Zomal Avenue,

Los Angeles, $C A$

90033, USA. Fax +1

3232267573.

\section{Patients}

742 patients (mean age 62 y, $68 \%$ women, $83 \%$ white) $\geqslant 50$ years of age with $\mathrm{OA}$ that had required non-steroidal anti-inflammatory drugs (NSAIDs) for $\geqslant 6$ months. Exclusion criteria were active ulcers; inflammatory bowel disease; previous upper gastrointestinal (GI) surgery; pyloric obstruction; erosive oesophagitis; abnormal serum creatinine levels or clearance; faecal occult blood; unstable medical conditions; history of cancer or cerebrovascular events; bleeding diathesis; or need for anticoagulants, ticlopidine, corticosteroids, or aspirin. The intention to treat analysis included $93 \%$ of the patients.

Table 1 Endoscopic gastroduodenal ulcers $\geqslant 3 \mathrm{~mm}$ with rofecoxib and placebo v ibuprofen for osteoarthritist

\begin{tabular}{llll} 
Comparison at 12 wk & Event rates & RRR (95\% Cl) & NNT (CI) \\
Rofecoxib, $25 \mathrm{mg} / \mathrm{d}, v$ ibuprofen & $4 \% v 25 \%$ & $85 \%(68$ to 93$)$ & 5 (3 to 7$)$ \\
\hline Rofecoxib, $50 \mathrm{mg} / \mathrm{d}, v$ ibuprofen & $7 \% v 25 \%$ & $73 \% 52$ to 85$)$ & $5(4$ to 9$)$ \\
\hline Placebo $v$ ibuprofen & $7 \% v 25 \%$ & $72 \%(49$ to 85$)$ & $5(4$ to 10$)$ \\
\hline
\end{tabular}

†Abbreviations defined in glossary; RRR, NNT, and $\mathrm{Cl}$ calculated using simple proportions with data provided by author.

\section{Intervention}

Patients were allocated to 16 to 24 weeks of rofecoxib, $25 \mathrm{mg} /$ day $(\mathrm{n}=195)$; rofecoxib, $50 \mathrm{mg} /$ day $(\mathrm{n}=186)$; ibuprofen, $2400 \mathrm{mg}$ /day $(\mathrm{n}=184)$; or placebo $(\mathrm{n}=177)$. Some other drugs were allowed (acetaminophen, non-NSAIDs, and an antacid [Gelusil]).

\section{Main outcome measures}

Endoscopic gastroduodenal ulcers $\geqslant 3 \mathrm{~mm}$ at 12 weeks (primary outcome) and 24 weeks (secondary outcome). A similar analysis was done with ulcers $\geqslant 5 \mathrm{~mm}$ at 12 and 24 weeks.

\section{Main results}

Patients in the rofecoxib groups and the placebo group had lower rates of endoscopic ulcers of both sizes than did patients in the ibuprofen group at 12 weeks (table 1); patients in both rofecoxib groups also had lower rates of endoscopic ulcers of both sizes at 24 weeks $(\mathrm{p}<0.001$ for comparisons with ibuprofen). The rofecoxib and placebo groups did not differ for any outcome. Rofecoxib and ibuprofen had similar efficacy.

\section{Conclusion}

Rofecoxib, 25 or $50 \mathrm{mg}$ /day, and placebo had lower rates of endoscopic gastroduodenal ulcers than did ibuprofen in patients with osteoarthritis.

*See glossary.

\section{COMMENTARY}

Most clinicians are aware of the discovery of 2 forms of cyclooxygenase (COX): COX-1, the constitutional form, produces prostaglandins involved in physiological functions; COX-2, an inducible form, produces inflammatory prostaglandins. The distinction between the 2 forms led to a search for drugs that inhibit COX-2, sparing COX-1, in the expectation that such agents would be free of the serious GI toxicity caused by conventional (non-selective) NSAIDs. The first 2 COX inhibitors, celecoxib and rofecoxib, enjoyed successful launches in North America before any publications were available to assess their effectiveness and toxicity. In late 1999, 4 full reports, including these studies by Laine and Simon and their colleagues and 2 others by Langman and Emery, ${ }^{2}$ were published; accompanying editorials raised doubts about the true value of these agents. ${ }^{3}{ }^{4}$ Overall, the 4 reports describe the experience of $>7000$ patients with RA or OA treated for 6 to 52 weeks (table 2 ).

Accepting that the COX-2 inhibitors are of similar efficacy to non-selective NSAIDs, the main clinical interest is in avoiding clinically important GI damage. During endoscopy, small and superficial ulcers that do not usually cause symptoms are frequently seen in the stomach and duodenum of patients taking conventional NSAIDs. The rates of these mainly non-clinical events with COX-2 inhibitors are approximately $25 \%$ of that with conventional NSAIDs. Physicians and patients are, however, more concerned with GI symptoms. Dyspepsia was reduced by only $2 \%$ to $3 \%$ with a COX-2 inhibitor. Although many patients have been studied in trials of COX-2 inhibitors, few instances of serious GI complications, such as bleeding or perforation, have occurred. The frequency of these potentially serious outcomes is approximately $1 \%$ with conventional NSAIDs; COX-2 inhibitors appear to provide a reduction of $0.5 \%$ to $1 \%$. This means that 100 to 200 "typical" patients will have to be treated (NNT) with a COX-2 NSAID instead of a conventional NSAID to avoid 1 additional serious complication. In low risk groups, the NNT for serious complications may be around $500{ }^{4}$

Many patients who are unable to tolerate several conventional NSAIDs will probably be switched to the newer agents, and some will benefit. Although not yet the topic of a published study, patients who are at high risk for serious GI complications (eg, a history of ulcers or GI bleeding) and who need to take an NSAID will probably be the group for whom COX-2 inhibitors will be the most cost effective. Considering the high cost of these new drugs and the widespread use of NSAIDs in most communities, the routine prescription of COX-2 inhibitors cannot be supported.

David Henry, MB, ChB

Patricia McGettigan, MD

University of Newcastle Newcastle, New South Wales, Australia

1 Langman MJ, Jensen DM, Watson DJ et al. Adverse upper gastrointestinal effects of rofecoxib compared with NSAIDs. JAMA 1999;282:1929-33.

2 Emery P, Zeidler H, Kvien TK, et al. Celecoxib versus diclofenac in long-term management of rheumatoid arthritis: randomised double-blind comparison. Lancet 1999;354:2106-11.

3 Beejay U, Wolfe MM. Cyclooxygenase 2 selective inhibitors: panacea or flash in the pan? Gastroenterology 1999;117:1002-14.

4 Peterson WL, Cryer B. COX-1-sparing NSAIDs-is the enthusiasm justified? JAMA 1999;282:1961-3. 